\title{
SHADOW REGION IMAGING ALGORITHM USING ARRAY ANTENNA BASED ON APERTURE SYNTHESIS OF MULTIPLE SCATTERED WAVES FOR UWB RADARS
}

\author{
Shouhei Kidera \\ Graduate School of Informatics, University of Electro-Communications, Tokyo, Japan \\ Email: kidera@ee.uec.ac.jp
}

\section{INTRODUCTION}

UWB (Ultra-wideband) radar with high range resolution creates various applications for near field sensing. It is applicable to non-contact measurement such as reflector antennas or aircraft bodies that have high-precision and specular surfaces, or spatial measurement for rescue or resource exploration robots that can identify a human body or materials even in darkness, smog or high-concentration gas. As a suitable imaging technique for these applications, we have already proposed the imaging algorithm called multiple scattered SAR (Synthetic Aperture Radar) [1]. This uses the principle that a multiple scattered signal includes additional independent information on the target points compared with information from a single scattered signal. In making use of the multiple scattered waves, this method significantly enhances the imagery range, including the region regarded as a shadow in the original SAR [2]. Furthermore, this method does not require a priori information of surroundings or target modeling, which is necessary for other techniques using multiple scattered waves [3, 4].

It has been demonstrated that while the former work enhanced the imagery range for several target cases, where the greater part of the target boundary still falls into a shadow, even when using the multiple scattering waves. This is because an antenna scanning observation is assumed for which the instantaneous aperture size often becomes insufficient to recognize a target shape, especially if it has a deep-set concave shape or a sharply-inclined boundary. To overcome the difficulty, this paper introduces an observation model using array antenna, where the instantaneous aperture size is enhanced. While this extension is quite simple, the proposed model greatly enhances the imagery range, despite the fact that the baseline of the antenna is the same as that in the antenna scanning model. The numerical simulation proves that the imagery range using the array antenna model expresses the target shape, which was never recognized by the former model.

\section{SYSTEM MODEL}

Fig. 1 illustrates the system model, where it assumes the 2-dimensional problem and TE mode waves, for simplicity. It also presumes that the target has high conductivity such as a metal,and an arbitrary shape with a clear boundary. The propagation speed of the radio wave $\mathrm{c}$ is assumed to be known constant. An omni-directional antenna is used, and the transmitting current

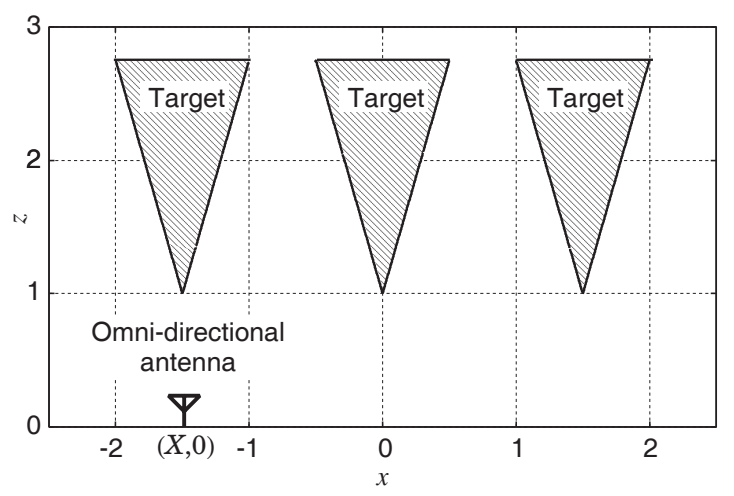

Fig. 1. System model. 

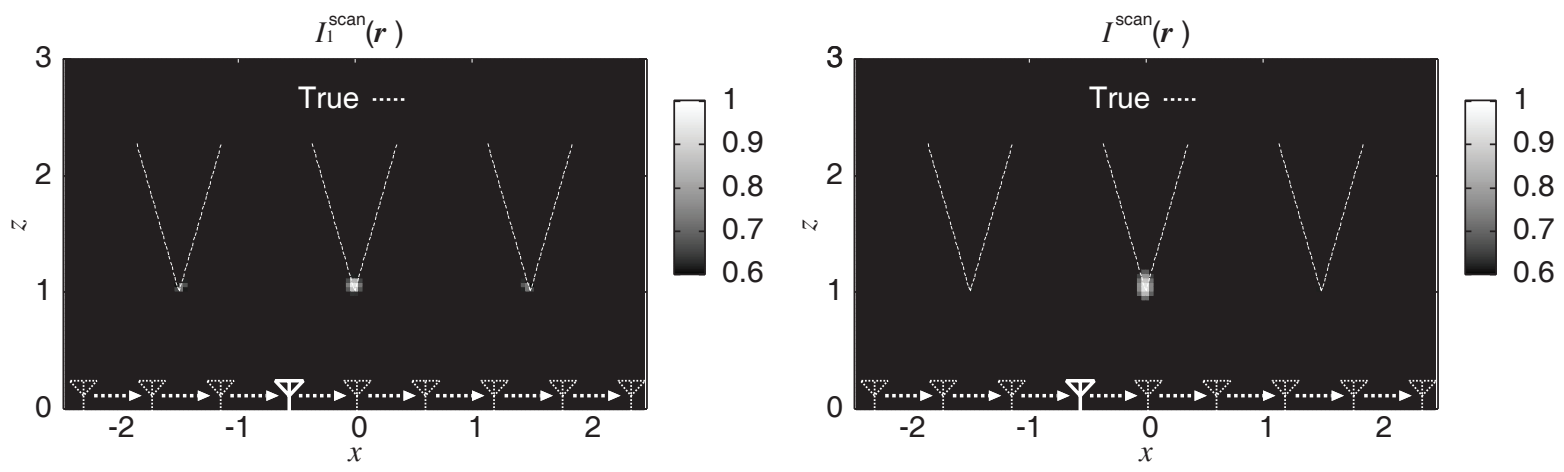

Fig. 2. The estimated images $I_{1}^{\text {scan }}(\boldsymbol{r})$ (left) and $I^{\text {scan }}(\boldsymbol{r})$ (right), where antenna scanning model is assumed.

is given by a mono-cycle pulse. The real space in which the target and antenna are located is expressed by the parameters $\boldsymbol{r}=(x, z)$, which are normalized by $\lambda$, the central wavelength of the pulse.

\section{CONVENTIONAL MODEL FOR MULTIPLE SCATTERED SAR}

The extended SAR algorithm using the double scattering signals has already been developed to enhance the imagery range, which becomes a shadow in the original SAR image [1]. This method assumes that a mono-static antenna is scanned along the $x$ axis, and $s(X, Z)$ is defined as the output of the Wiener filter at the antenna location $(X, 0)$, where $Z=c t /(2 \lambda)$ is expressed by the time $t$. The procedure creating $s(X, Z)$ is detailed in [5]. This method is based on the simple principle that, "a double scattered wave propagates a different path from that of a single scattered one, and this wave often includes significant information on two reflection points on the target boundaries". The suitable use of multiple scattered signals is promising as shadow region imaging. It calculates the image migrated by double scattered signals as

$$
I_{2}^{\text {scan }}(\boldsymbol{r})=-\int_{\boldsymbol{r}^{\prime} \in R} \int_{X \in \Gamma} I_{1}^{\text {scan }}\left(\boldsymbol{r}^{\prime}\right) s\left(X, d\left(\boldsymbol{r}, \boldsymbol{r}^{\prime}, X, X\right) / 2\right) \mathrm{d} X \mathrm{~d} x^{\prime} \mathrm{d} z^{\prime},
$$

where $\Gamma$ is the scanning range of the antenna, $\boldsymbol{r}^{\prime}=\left(x^{\prime}, z^{\prime}\right)$ is defined, $R$ is the region of the real space, and $d\left(\boldsymbol{r}, \boldsymbol{r}^{\prime}, X, X^{\prime}\right)=$ $\sqrt{(x-X)^{2}+z^{2}}+\sqrt{\left(x^{\prime}-X^{\prime}\right)^{2}+z^{\prime 2}}+\sqrt{\left(x-x^{\prime}\right)^{2}+\left(z-z^{\prime}\right)^{2}}$ holds. The minus sign of the right term in Eq. (1) creates a positive image focused by double scattered waves, that have an anti-phase relationship compared with single scattered waves. The initial image $I_{1}^{\text {scan }}(\boldsymbol{r})$ is defined as the original SAR image as

$$
I_{1}^{\text {scan }}(\boldsymbol{r})=\int_{X \in \Gamma} s(X, d(\boldsymbol{r}, \boldsymbol{r}, X, X) / 2) \mathrm{d} X,
$$

Eq.(1) expresses the aperture synthesis of the received signals by considering only a double scattered path. The final image is defined as $I^{\text {scan }}(\boldsymbol{r})=w I_{1}^{\text {scan }}(\boldsymbol{r}) H\left(I_{1}^{\text {scan }}(\boldsymbol{r})\right)+(1-w) I_{2}^{\text {scan }}(\boldsymbol{r}) H\left(I_{2}^{\text {scan }}(\boldsymbol{r})\right)$, where $H(*)$ is the Heaviside function, and $w$ is empirically determined. This method directly enhances the imagery range with only a single observation, and does not require any priori information on surroundings, or target modeling, which are substantial advantages for the other algorithms [3, 4].

An example of this method is presented as follows. The received signals are calculated by the FDTD method, and obtained at 26 locations in the range, $-2.5 \leq X \leq 2.5$. The target boundary is assumed as shown in Fig. 1. The left and right hand sides of Fig. 2 show $I_{1}^{\text {scan }}(\boldsymbol{r})$ and $I^{\text {scan }}(\boldsymbol{r})$, respectively. $w=0.3$ is set. Each image is normalized by its maximum value. $I_{1}^{\text {scan }}(\boldsymbol{r})$ expresses only the convex edges of the triangle boundary because only single scattered signals are used for imaging. In addition, the right hand side of Fig. 2 shows that the triangle side of the target is still not reconstructed, despite the use of double scattered waves. This is because the double scattered signals, that propagate through the sharp inclined side of the triangles, are not observed for any antenna location. The above result suggests that, to enhance the range of the radar imagery, a large instantaneous aperture size is necessary.

\section{PROPOSED MODEL FOR MULTIPLE SCATTERED SAR}

To outperform the former algorithm, we propose to use the array antenna model for the multiple scattered SAR, which can enhance an instantaneous aperture size. The left and right hand sides of Fig. 3 show the visible points in the use of antenna 

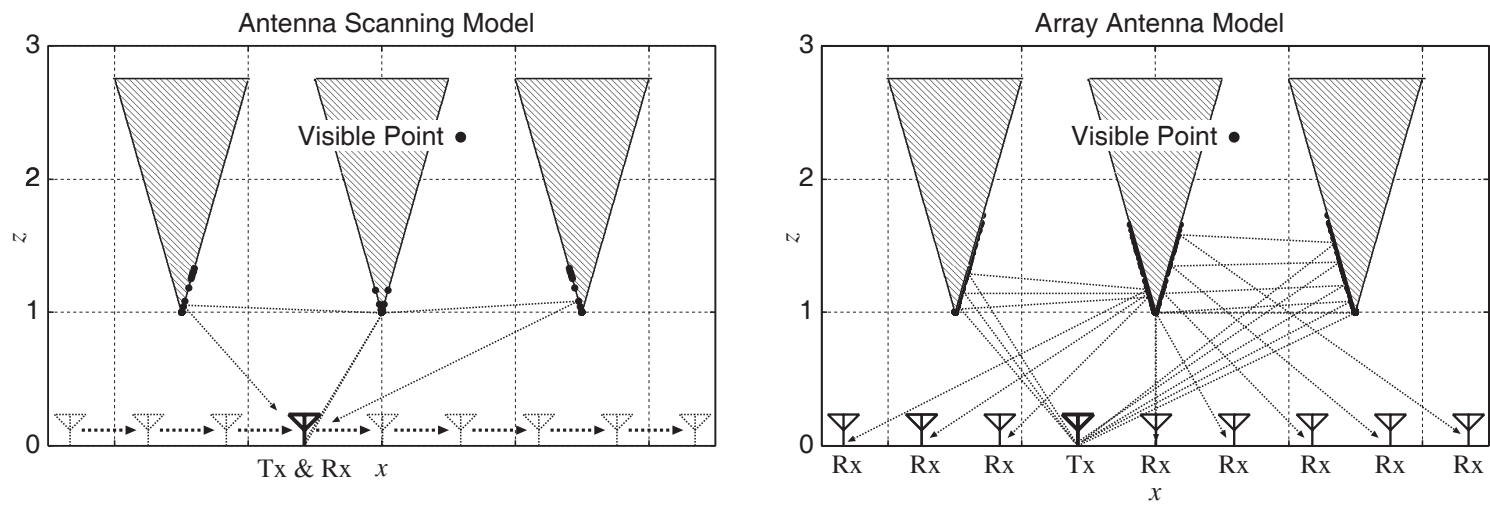

Fig. 3. Visible points with double scattered waves in antenna scanning (left) and array antenna models (right).

scanning and array antenna models, respectively. The numbers of both scanning samples or array antenna are 26 for $-2.5 \leq$ $x \leq 2.5$. Each visible point is calculated using the geometrical optics, considering that the propagation path is secluded from other targets. In the scanning model as shown in the left side of Fig. 3, there are a few points around the lower side of triangle boundaries, but the most of the visible points are concentrated on the edges. It is confirmed that the locations of these points does not change significantly, even if the number of scanning sample increases. Conversely, in the array antenna model, the number of the visible points around each triangle's side remarkably increases, and the central target shape can be identified as the part of triangle, despite an aperture size that is equivalent to the aperture in the previous model. This is because the combination of the transmitting and receiving antennas creates different scattering paths, and increases the number of independent target points. This reveals that the extension for the array model offers a substantial improvement, especially for the multiple scattered SAR algorithm.

Here, the transmitting and receiving antenna locations are defined as $\left(X_{\mathrm{T}}, 0\right)$ and $\left(X_{\mathrm{R}}, 0\right)$, respectively. In each combination of $X_{\mathrm{T}}$ and $X_{\mathrm{R}}$, the output of the Wiener filter is obtained as $s\left(X_{\mathrm{T}}, X_{\mathrm{R}}, Z\right)$. The previous research is readily extended to the bi-static model, and the estimated image with the array antenna model $I_{2}^{\text {array }}(\boldsymbol{r})$ is calculated as,

$$
I_{2}^{\text {array }}(\boldsymbol{r})=-\int_{\boldsymbol{r}^{\prime} \in R} \int_{X_{\mathrm{R}} \in \Gamma} \int_{X_{\mathrm{T}} \in \Gamma} I_{1}^{\text {array }}\left(\boldsymbol{r}^{\prime}\right) s\left(X_{\mathrm{T}}, X_{\mathrm{R}}, d\left(\boldsymbol{r}, \boldsymbol{r}^{\prime}, X_{\mathrm{T}}, X_{\mathrm{R}}\right) / 2\right) \mathrm{d} X_{\mathrm{T}} \mathrm{d} X_{\mathrm{R}} \mathrm{d} x^{\prime} \mathrm{d} z^{\prime},
$$

where $I_{1}^{\text {array }}(\boldsymbol{r})$ is defined as,

$$
I_{1}^{\text {array }}(\boldsymbol{r})=\int_{X_{\mathrm{T}} \in \Gamma} \int_{X_{\mathrm{R}} \in \Gamma} s\left(X_{\mathrm{T}}, X_{\mathrm{R}}, d\left(\boldsymbol{r}, \boldsymbol{r}, X_{\mathrm{T}}, X_{\mathrm{R}}\right) / 2\right) \mathrm{d} X_{\mathrm{R}} \mathrm{d} X_{\mathrm{T}} .
$$

The final image is defined as $I^{\text {array }}(\boldsymbol{r})=w I_{1}^{\text {array }}(\boldsymbol{r}) H\left(I_{1}^{\text {array }}(\boldsymbol{r})\right)+(1-w) I_{2}^{\text {array }}(\boldsymbol{r}) H\left(I_{2}^{\text {array }}(\boldsymbol{r})\right)$. This array model significantly enhances the instantaneous aperture size. That is, it expands a visible range in spite of the fact that the baseline lengths of both the conventional and proposed models are the same.

\section{PERFORMANCE EVALUATION}

This section presents the example used in the proposed model. The left and right hand sides of Fig. 4 show $I_{1}^{\text {array }}(\boldsymbol{r})$ and $I^{\text {array }}(\boldsymbol{r})$ for the triangular objects, respectively. The number of array antenna is 26, assuming the same baseline as in Fig. 2 . $w=0.3$ is set. The left hand side of Fig. 4 indicates that the image obtained by single scattered SAR $I_{1}^{\text {array }}(\boldsymbol{r})$ does not enhance the imagery range, entirely, even if the instantaneous aperture size is enhanced. This is because it uses only the single scattered wave for imaging. On the contrary, the right hand side of Fig. 4 reveals that the triangular side of the target is reconstructed, and offers a substantial image identifying the triangular shapes. This is because the array observation increases the number of double scattered signals, which includes independent information on the target boundary, while the enhanced instantaneous aperture size improves the imagery range.

It confirms that the image obtained by the proposed method remains accurate in a noisy situation, where the S/N of the double scattered signal is higher than $20 \mathrm{~dB}$. In addition, this method requires the quadruple integration of the received signals in Eq. (3), which requires about 20 minutes for the calculation using a Xeon $3.2 \mathrm{GHz}$ processor. Thus an acceleration in the imaging speed is also required to use the extended method for 3-dimensional problems. 

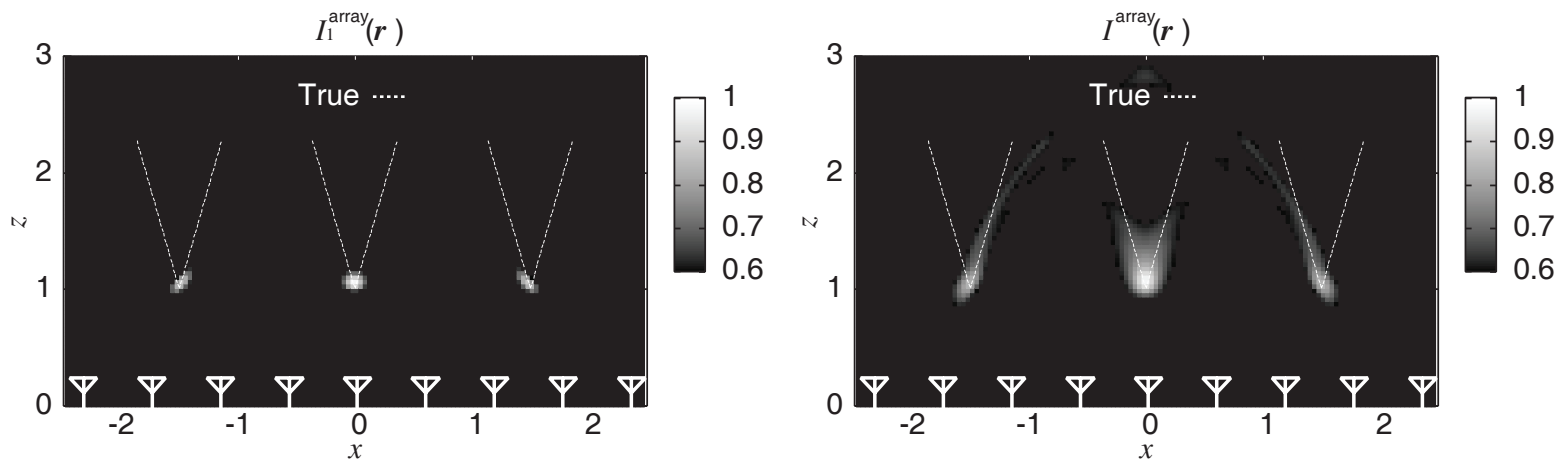

Fig. 4. $I_{1}^{\text {scan }}(\boldsymbol{r})$ (left) and $I^{\text {scan }}(\boldsymbol{r})($ right) in array antenna model.

\section{CONCLUSION}

This paper proposed a shadow imaging algorithm based on aperture synthesis of double scattered waves using an array antenna model. The previous work is extended to the array antenna observation, where a bi-static model of the multiple scattered SAR is introduced. Although this extension is simple and not novel in itself, the imagery range is substantially improved with the larger instantaneous aperture size, especially when using the multiple scattered SAR method. The result of the numerical simulation successfully proves that the proposed model makes shadow region visible for one of the most difficult target cases, despite the fact that the baseline length is the same for both conventional and proposed models.

\section{REFERENCES}

[1] S. Kidera, T. Sakamoto and T. Sato, " Experimental study of shadow region imaging algorithm with multiple scattered waves for UWB radars," Proc of Progress in Electromagnetics Research Symposium (PIERS), Vol. 5, No. 4, pp. 393-396, Aug, 2009.

[2] D. L. Mensa, G. Heidbreder and G. Wade, "Aperture Synthesis by Object Rotation in Coherent Imaging," IEEE Trans. Nuclear Science., vol. 27, no. 2, pp. 989-998, Apr, 1980.

[3] S. K. Lehmanm and A. J. Devaney, "Transmission mode time-reversal super-resolution imaging," Acoust. Soc. Am., 113 (5), May, 2003.

[4] J. M. F. Moura, and Y. Jin, “Detection by Time Reversal: Single Antenna,” IEEE Trans. on Signal Process., vol. 55, no. 1, pp. 187-201, Jan, 2007.

[5] S. Kidera, T. Sakamoto and T. Sato, "Accurate UWB Radar 3-D Imaging Algorithm for Complex Boundary without Range Points Connections," IEEE Trans. Geosci. Remote Sens., vol. 48, no. 4, pp. 1993-2004, Apr., 2010. 